\title{
Some Results on Lipschitzian Stochastic Differential Equations by Dirichlet Forms Methods.
}

\author{
Nicolas BOULEAU \\ ENPC \\ La Courtine \\ 93167 Noisy-le-Grand cedex
}

France
Francis HIRSCH

ENS Cachan

61 Av. Président Wilson

94230 Cachan

France

Since the impulse given by $\mathrm{P}$. Malliavin, the stochastic calculus of variations has been mainly applied to stochastic differential equations with $C^{\infty}$ coefficients, see Ocone [O1] for a comprehensive exposition.

But it is also important for applications to get regularity results for solutions of SDE with less smooth coefficients and in particular under Lipschitz hypotheses which are, in dimension greater than one, the most natural hypotheses of existence and uniqueness of solutions.

The celebrated integration by parts method cannot apparently be extended beyond the case of functionals in the domain $\mathcal{D} L$ of the Ornstein-Uhlenbeck operator $\left(\mathbb{D}_{2,2}\right.$ with the notations of Watanabe [W1]), so that the regularity of solutions of Lipschitzian SDE must come from specific technics. Especially well adapted are Dirichlet forms methods which allow to exploit intensively the fact that Lipschitz functions operate on $\mathbb{D}_{2,1}=\mathcal{D} \sqrt{-L}$.

We give here an account of results already obtained in this direction by Dirichlet forms methods and we present in details a new example which gives rise to an extension of the stochastic calculus. The first part introduces the framework of the Dirichlet space related to the Ornstein-Uhlenbeck semigroup on the Wiener space and recalls the absolute continuity criterion (cf [B-H1] [B-H2]) for functionals in $\mathbb{D}_{2,1}$ or $\mathbb{D}_{2,1}^{\text {loc }}$ and some consequences on Lipschitz SDE.

The second part is devoted to the regularity of solutions of Lipschitz SDE with respect to initial data. It is shown that the solution is differentiable in a slightly weakened sense. That gives for example the following simple result: under these hypotheses, if the initial variable $X_{0}$ has a density, then $X_{t}$ has a density for all $t$. 
After recalling the definition of the capacity associated with the OrnsteinUhlenbeck Dirichlet form, it is shown in the third part, that the solutions of Lipschitz SDE can be refined, by taking quasi-continuous versions for each $t$, into processes with continuous paths outside a polar set and unique up to a quasievanescent set. The main tool here is an extension of the Kolmogorov theorem on existence of continuous versions to the case where the measure is changed to a capacity.

This allows to study the solutions of Lipschitz SDE under measures which do not charge polar sets. In the last part, using Wiener chaos decompositions of positive distributions, we show that this property allows an extension of the stochastic calculus by constructing a finite energy measure singular with respect to the Wiener measure and for which the coordinates do not build a semimartingale. This answers a conjecture formulated in [B-H4].

\section{The structure of Dirichlet space on the Wiener space associated with the Ornstein - Uhlenbeck semigroup.}

The Wiener space

$$
\Omega=\left\{\omega \in \mathcal{C}\left(\mathbb{R}_{+}, \mathbb{R}^{d}\right) ; \omega(0)=0\right\}
$$

is equipped with the topology of uniform convergence on compact sets, with its Borelian $\sigma$-algebra and with the Wiener measure $m$ which makes the coordinates a standard Brownian motion. $\mathcal{F}$ denotes the $m$-completed $\sigma$-algebra of $\sigma\left(B_{t} ; t \in\right.$ $\left.\mathbb{R}_{+}\right)$, and $\mathcal{F}_{t}$ the $\mathcal{F}$ - $m$-completed $\sigma$-algebra of $\sigma\left(B_{s} ; s \leq t\right)$.

We consider on $L^{2}(m)$ the Ornstein-Uhlenbeck semigroup $P_{t}$ a strongly continuous symmetric Markovian semigroup characterized by

$$
\begin{gathered}
P_{t}\left[\exp \left\{\int h(s) \cdot d B_{s}-\frac{1}{2}\|h\|^{2}\right\}\right]=\exp \left\{e^{-t / 2} \int h(s) \cdot d B_{s}-\frac{1}{2}\left\|e^{-t / 2} h\right\|^{2}\right\} \\
\forall h \in H=L^{2}\left(\mathbb{R}_{+}, \mathbb{R}^{d}\right) .
\end{gathered}
$$

The self-adjoint operator generator of $P_{t}$ is denoted by $L$. It corresponds to $P_{t}$ (cf [B-H1]) a Dirichlet form with domain

$$
\mathbb{D}=\mathcal{D}(\sqrt{-L})
$$

given by

$$
((u, u))=\|\sqrt{-L} u\|_{L^{2}(m)}^{2} .
$$

That means that the space $\mathbb{D}$ with the norm $\left(\|u\|_{L^{2}(m)}^{2}+((u, u))\right)^{1 / 2}$ is complete and that normal contractions operate: For all $u \in \mathbb{D}$, for all measurable $v$ such 
that $\forall \omega|v(\omega)| \leq|u(\omega)|$ and $\forall \omega, \omega^{\prime}\left|v(\omega)-v\left(\omega^{\prime}\right)\right| \leq\left|u(\omega)-u\left(\omega^{\prime}\right)\right|$, one has $v \in \mathbb{D}$ and $((v, v)) \leq((u, u))$.

This Dirichlet form is local ([B-H1] p239) and possesses a carré-du-champ operator, i.e. a symmetric bilinear continuous map $\Gamma$ from $\mathbb{D} \times \mathbb{D}$ into $L^{1}(m)$ such that $\forall u, v \in \mathbb{D} \cap L^{\infty}(m)$,

$$
2((u v, u))-\left(\left(v, u^{2}\right)\right)=\int v \Gamma(u, u) d m .
$$

This Dirichlet structure $(\Omega, \mathcal{F}, m ;((.,)),. \mathbb{D})$ is related to the Sobolev spaces which are classically defined on the Wiener space in the following way:

Let $\mathbb{D}_{p, s}, p \in(1, \infty), s \in \mathbb{R}$ the closure of the linear space generated by polynomials in continuous linear forms on $\Omega$ for the norm

$$
\|F\|_{p, s}=\left\|(I-L)^{s / 2} F\right\|_{p}
$$

(cf [W1]). Then $\mathbb{D}=\mathbb{D}_{2,1}$ and $\|F\|_{L^{2}}^{2}+((F, F))=\|F\|_{2,1}^{2}$.

Let

$$
\xi_{n}=\int_{0}^{t} \dot{\xi}_{n}(s) d s
$$

where $\dot{\xi}_{n}$ is a complete orthonormal system of $H=L^{2}\left(\mathbb{R}_{+}, \mathbb{R}^{d}\right)$, then for all $u \in \mathbb{D}$ the following limit exists in probability (cf [B-H2])

$$
\nabla_{\xi_{n}}(u)(\omega)=\lim _{t \downarrow 0} t^{-1}\left[u\left(\omega+t \xi_{n}\right)-u(\omega)\right]
$$

and one has

$$
\Gamma(u, u)=\sum\left(\nabla_{\xi_{n}} u\right)^{2} .
$$

The derivation operator $D$ (cf [W1]) which can be defined by

$$
D u=\sum_{n}\left(\nabla_{\xi_{n}} u\right) \dot{\xi}_{n}
$$

and which is continuous from $\mathbb{D}=\mathbb{D}_{2,1}$ into $L^{2}(\Omega, H)$ is related to the carré-duchamp operator $\Gamma$ by

$$
\Gamma(u, u)=<D u, D u>_{H}, \forall u \in \mathbb{D}_{2,1} .
$$

This relation between the carre-du-champ operator and the derivations in the directions of Cameron-Martin vectors $(\xi \in \Omega$ s.t. $\dot{\xi} \in H)$ allows, by an extension of the co-area formula of Federer [F1], to obtain the following absolute continuity criterion:

Proposition 1 . Let $u=\left(u_{1}, \ldots, u_{n}\right) \in\left(\mathbb{D}_{2,1}\right)^{n}$, the image by $u$ of the measure

$$
\operatorname{det}\left[\Gamma\left(u, u^{*}\right)\right] . m
$$

is absolutely continuous with respect to the Lebesgue measure on $\mathbb{R}^{n}$. 
When $n=1$, this result is true for any local Dirichlet space (cf [B-H1]) and also for the local energy part in any Dirichlet space on a locally compact space (cf [B1]).

In fact proposition 1 remains valid for $u$ in $\left(\mathbb{D}_{2,1}^{l o c}\right)^{n}$ defined by

$$
\mathbb{D}_{2,1}^{\text {loc }}=\left\{u: \Omega \rightarrow \mathbb{R} ; \exists \Omega_{n} \in \mathcal{F}, \Omega_{n} \uparrow \Omega, \forall n \exists u_{n} \in \mathbb{D}_{2,1}, u=u_{n} \text { on } \Omega_{n}\right\}
$$

and for $u \in \mathbb{D}_{2,1}^{l o c}, \Gamma(u, u)$ depends only on $u$.

An important application of the extension of Dirichlet forms methods to the case of the Wiener space is the study of stochastic differential equations. Let us specify the Lipschitz hypotheses which will be in force in the sequel:

Two Borelian functions $\sigma, b$ are given

$$
\begin{gathered}
\sigma: \mathbb{R}_{+} \times \mathbb{R}^{n} \longrightarrow \mathbb{R}^{n \times d} \\
b: \mathbb{R}_{+} \times \mathbb{R}^{n} \longrightarrow \mathbb{R}^{n}
\end{gathered}
$$

and there exists $K: \mathbb{R}_{+} \rightarrow \mathbb{R}_{+}$such that

$$
\begin{gathered}
\forall T \in \mathbb{R}_{+}, \forall t \in[0, T], \forall x, y \in \mathbb{R}^{n} \\
|\sigma(t, x)| \vee|b(t, x)| \leq K(T)(1+|x|) \\
|\sigma(t, x)-\sigma(t, y)| \vee|b(t, x)-b(t, y)| \leq K(T)(|x-y|) .
\end{gathered}
$$

One is concerned by the equation

$$
d X_{t}=\sigma\left(t, X_{t}\right) \cdot d B_{t}+b\left(t, X_{t}\right) d t
$$

From the fact that contractions hence Lipschitz functions operate on the Dirichlet space, it follows ( $\mathrm{cf}[\mathrm{B}-\mathrm{H} 1]$ [B-H2]) that the solution of $(1)$ is such that the map $t \rightarrow X_{t}$ is continuous from $\mathbb{R}_{+}$into $\left(\mathbb{D}_{2,1}\right)^{n}$ and by writing down a stochastic differential equation satisfied by the matrix $\Gamma\left(X_{t}, X_{t}^{*}\right)$ it is possible to bring out conditions under which $X_{t}$ has a density by application of proposition 1 .

For example if $A_{k}=\{(t, y): \sigma(t, y)$ is of rank $k\}$ and if $T_{k}$ is the essential beginning of $A_{k}$ for $\left(X_{t}\right)_{t \geq 0}$, one gets that for $t$ such that $m\left(\left\{t>T_{k}\right\}\right)>0$ and for almost all subspace $V$ of $\mathbb{R}^{n}$ of dimension $k$, the projection of $X_{t}$ on $V$, knowing $\left\{t>T_{k}\right\}$, has a density with respect to the Lebesgue measure on $V$.

\section{Regularity of solutions of Lipschitz SDE with respect to the initial data}

Under these Lipschitz hypotheses, it is known ( $\mathrm{cf}[\mathrm{K} 2]$ ) that there exists a version $\left(X_{t}^{x}(\omega)\right)_{(t, x) \in \mathbb{R}_{+} \times \mathbb{R}^{n}}$ of the solution of (1) starting at $x$, such that for almost all $\omega$ 
the map $(t, x) \rightarrow X_{t}^{x}(\omega)$ is continuous and for all $t \geq 0 x \rightarrow X_{t}^{x}(\omega)$ is an onto homeomorphism of $\mathbb{R}^{n}$.

If it is supposed further that $\sigma$ and $b$ are $C^{1, \alpha}$ with respect to $x$ then $x \rightarrow X_{t}^{x}(\omega)$ is an onto $C^{1}$ - diffeomorphism.

Under the only Lipschitz hypotheses, $X_{t}^{x}$ is of course not $C^{1}$ with respect to $x$ in general, but it is possible to show that the Jacobian $\frac{\partial}{\partial x}\left(X_{t}^{x}(\omega)\right)$ exists in a weakened sense and satisfies a SDE which can be written explicitly.

For this, consider the space $\tilde{\Omega}=\mathbb{R}^{n} \times \Omega$ equipped with the probability $\tilde{m}=$ $h(x) d x \times m$ where $\mathrm{m}$ is the Wiener measure on $\Omega$ and $h$ a strictly positive continuous function such that $\int h(x) d x=1, \int|x|^{2} h(x) d x<+\infty$. The $\sigma$-algebras generated by applications $B_{s}, s \leq t$ and completed for $\tilde{m}$ are denoted by $\tilde{\mathcal{F}}_{t}$.

$(\tilde{\Omega}, \tilde{m})$ gets a natural Dirichlet form associated with the derivations in directions given by the canonical basis of $\mathbb{R}^{n}$. In other words the tool is here the form

$$
((u, v))=\int \sum_{i=1}^{n}\left(\frac{\partial u}{\partial x_{i}} \frac{\partial v}{\partial x_{i}}\right) d \tilde{m}
$$

with domain and operators $\partial / \partial x_{i}$ suitably defined.

We denote by $\left(\tilde{X}_{t}\right)_{t \geq 0}$ [resp. $\left.\left(\tilde{B}_{t}\right)_{t \geq 0}\right]$ the class of the process $\left(X_{t}^{x}\right)_{t \geq 0}$ [resp. of the Brownian motion $\left(B_{t}\right)_{t \geq 0}$ ] enlarged up to $\tilde{m}$-evanescent sets.

Proposition 2 . Under the Lipschitz hypotheses,

a) for $m$-almost all $\omega, \forall t \geq 0, X_{t}^{\bullet}(\omega) \in\left(H_{l o c}^{1}\left(\mathbb{R}^{n}\right)\right)^{n}$

b) there exists a process $\left(M_{t}(x, \omega)\right)_{t \geq 0},\left(\tilde{\mathcal{F}}_{t}\right)$-adapted, with continuous paths and values in $G L_{n}(\mathbb{R})$, such that

for m-almost all $\omega, \forall t \geq 0,\left[\frac{\partial}{\partial x}\left(X_{t}^{x}(\omega)\right)=M_{t}(x, \omega) d x\right.$-a.e. $]$

c) let $\sigma^{\prime}$ and $b^{\prime}$ be fixed Borelian versions of the derivatives $\frac{\partial}{\partial x} \sigma(t, x)$ and $\frac{\partial}{\partial x} b(t, x)$, then $M$ is the unique $\left(\tilde{\mathcal{F}}_{t}\right)$-adapted continuous solution, defined up to an $\tilde{m}$-evanescent set, of the $S D E$

$$
\left\{\begin{array}{l}
d M_{t}=\left[\sigma^{\prime}\left(t, \tilde{X}_{t}\right) \cdot M_{t}\right] d B_{t}+\left[b^{\prime}\left(t, \tilde{X}_{t}\right) \cdot M_{t}\right] d t \\
M_{0}=I
\end{array}\right.
$$

It follows from this proposition and from a variant of proposition 1 applied to the Dirichlet structure on $\tilde{\Omega}$ explained above that the equation (1) with initial value a random variable independent of $\left(B_{t}\right)$ possessing a density, has a solution which admits a density for all $t \geq 0$. This was known, apparently, in dimension greater than one, only under $C^{1, \alpha}$ hypotheses.

In dimension 1 , there is an explicit solution : if we write as before $\sigma_{i}^{\prime}, b^{\prime}$ for fixed Borelian versions of the derivatives of $\sigma$ and $b$ with respect to $x$, the process

$$
Y_{t}^{x}=\exp \left\{\sum_{i=1}^{d}\left(\int_{0}^{t} \sigma_{i}^{\prime}\left(s, X_{t}^{x}\right) d B_{s}^{i}-\frac{1}{2} \int_{0}^{t}\left[\sigma_{i}^{\prime}\left(s, X_{t}^{x}\right)\right]^{2} d s\right)+\int_{0}^{t} b^{\prime}\left(s, X_{t}^{x}\right) d s\right\}
$$


is such that for $m$-almost all $\omega$,

$$
\forall \alpha, \beta \in \mathbb{R}, \forall t \geq 0, \quad X_{t}^{\beta}(\omega)-X_{t}^{\alpha}(\omega)=\int_{\alpha}^{\beta} Y_{t}^{x}(\omega) d x
$$

\section{Regularity, up to a polar set, of the solutions and their flows.}

The Dirichlet form on the Wiener space associated with the Ornstein-Uhlenbeck operator makes it possible to look at properties of the Brownian motion satisfied up to a zero capacity set (cf [F3], [K1],[S2]).

We study here, from this point of view, properties of solutions of Lipschitz SDE. A work in the same spirit was done independently by J. Ren (cf [R1]) for equations with $C^{\infty}$-coefficients and with thin sets associated with $C_{p, s}$-capacities (cf [M1]).

We denote by $C$ the capacity associated with the Ornstein-Uhlenbeck Dirichlet form. It is defined by

$$
C(G)=\inf \left\{\|u\|_{2,1}^{2} ; u \in \mathbb{D}, u \geq 1 m \text { - a.e. on } G\right\}
$$

if $G$ is an open set, and by

$$
C(G)=\inf \{C(G) G \text { open and } G \supset A\}
$$

if $A \in \mathcal{F}$.

If $C(A)=0, A$ is said to be a polar set.

$f: \Omega \rightarrow \mathbb{R}$ is said to be quasi-continuous with respect to the capacity $C$ if $\forall \epsilon>0, \exists \Omega_{\epsilon}$ open with $C\left(\Omega_{\epsilon}\right)<\epsilon$ such that $f$ restricted to the complementary $\Omega_{\epsilon}^{c}$ of $\Omega_{\epsilon}$ is continuous.

Two processes $\left(u_{\lambda}\right)_{\lambda \in \Lambda},\left(v_{\lambda}\right)_{\lambda \in \Lambda}$ defined on $\Omega$ are said to be C-indistinguishable if there exists a polar set $A$ such that $\forall \omega \notin A, \forall \lambda \in \Lambda, u_{\lambda}(\omega)=v_{\lambda}(\omega)$.

Under the Lipschitz hypotheses, we know that the solution $X_{t}^{x}(\omega)$ of equation (1) starting at $x$ is such that for fixed $t, x, X_{t}^{x} \in \mathbb{D}_{2,1}^{n}$. It follows that this random variable admits a quasi-continuous version defined up to a polar set. The following extension of the Kolmogorov theorem gives conditions under which it is possible to put these quasi-continuous versions together to get a continuous process outside a polar set.

Proposition 3 . Let $\left(u_{x}\right)_{x \in \mathbb{R}^{r}}$ be a family of elements of $\mathbb{D}$ and $p, \alpha_{1}, \ldots, \alpha_{r}$ stricly positive real numbers. Suppose the following conditions hold 


$$
\begin{array}{cc}
\diamond & \sum_{i=1}^{r} \frac{1}{\alpha_{i}}<1 \\
\diamond & \forall x, y \in \mathbb{R}^{r}\left|u_{x}-u_{y}\right|^{p} \in \mathbb{D} \\
\diamond & \exists L: \mathbb{R}_{+} \rightarrow \mathbb{R}_{+}, \forall R>0, \forall x, y \in \mathbb{R}^{r} \\
& |x| \vee|y| \leq \mathbb{R} \Longrightarrow\left\|\left|u_{x}-u_{y}\right|^{p}\right\|_{2,1}^{2} \leq L(R) \sum_{i=1}^{r}\left|x_{i}-y_{i}\right|^{\alpha_{i}}
\end{array}
$$

Then there exists a family $\left(v_{x}\right)_{x \in \mathbf{R}^{r}}$ such that

i) $x \rightarrow v_{x}(\omega)$ is continuous

ii) for all $x v_{x}$ is a quasi-continuous version of $u_{x}$.

The family $\left(v_{x}\right)$ is unique up to $C$-indistinguishability and the following uniformity properties hold:

There exist open sets $\left(\Omega_{\epsilon}\right)_{\epsilon>0}$ with compact complementary $\Omega_{\epsilon}^{c}$ such that

a) $\forall \epsilon>0, C\left(\Omega_{\epsilon}\right)<\epsilon$ and the map $(x, \omega) \in \mathbb{R}^{r} \times \Omega_{\epsilon}^{c} \rightarrow v_{x}(\omega) \in \mathbb{R}$ is continuous

b) $\forall \beta_{i}, 0<\beta_{i}<\alpha_{i}\left(1-\sum_{j=1}^{r} 1 / \alpha_{i}\right) / 2 p \quad i=1, \ldots, r$

$\exists K>0, \forall \epsilon>0, \forall R>0, \exists \eta>0$,

$\left(\omega \in \Omega_{\epsilon}^{c},|x| \vee|y| \leq \mathbb{R},|x-y| \leq \eta\right) \Rightarrow\left|v_{x}(\omega)-v_{y}(\omega)\right| \leq K \sum_{i=1}^{r}\left|x_{i}-y_{i}\right|^{\beta_{i}}$.

This criterion allows to show that under the Lipschitz hypotheses and for a given fixed initial condition $x \in \mathbb{R}^{n}$, the solution $X_{t}^{x}$ of equation (1) can be made more accurate into a process $\left(\tilde{X}_{t}\right)_{t \geq 0}$ unique up to $C$-indistinguishability such that

i) $t \rightarrow \tilde{X}_{t}$ is continuous,

ii) for all $t \tilde{X}_{t}$ is quasi-continuous and $\tilde{X}_{t}=X_{t}^{x} m-$ a.s..

This result has been extended, by using a Banach valued space $\mathbb{D}_{2,1}$ by $\mathrm{D}$. Feyel and A. de la Pradelle [F2] to the case of Ito processes of the form

$$
X_{t}=\int_{0}^{t} \alpha_{s} . d B_{s}+\int_{0}^{t} \beta_{s} . d s
$$

with $\alpha, \beta \in L^{2}\left(\mathbb{R}_{+}, \mathbb{D}\right)$ and adapted.

The previous criterion of Kolmogorov type, allows also to obtain a quasicontinuous version $\tilde{X}_{t}^{x}$ of $X_{t}^{x}$ which is for $\omega$ outside a polar set, continuous in $(t, x)$ and an onto homeomorphism with respect to $x$; but for this $C^{1, \alpha}$-hypotheses in $x$ are needed for $\sigma$ and $b$ (cf [B-H4]).

With $C^{2, \alpha}$-hypotheses, the differentiability with respect to $x$ of the flow is obtained with a quasi-continuous regular Jacobian matrix $\frac{\partial}{\partial x} \tilde{X}_{t}^{x}(\omega)$ continuously depending on $(t, x)$ for $\omega$ outside a polar set (see [B-H4] theorems V.1 and V.2 for more precise results). 


\section{Stochastic calculus under a probability which does not charge polar sets}

We keep in the sequel the preceding globally Lipschitz hypotheses and look at the solution of

$$
X_{t}=x+\int_{0}^{t} \sigma\left(s, X_{s}\right) \cdot d B_{s}+\int_{0}^{t} b\left(s, X_{s}\right) d s
$$

which is continuous in $t$, quasi-continuous in $\omega$ and unique up to $C$-indistinguishability.

This process is well defined under any probability measure on the Wiener space which does not charge polar sets.

A. The first case is when the right hand side of (2) also makes sense under such a measure $\nu$.

To be precise with the changes of measure we introduce the $\sigma$-fields $\mathcal{F}_{t}^{0}=$ $\sigma\left(B_{s}, s \leq t\right)$ without any completion.

It can be shown (cf [B-H4]) that there exists an $\left(\mathcal{F}_{t}^{0}\right)$-adapted solution, $\tilde{X}_{t}$, of (2) such that, for fixed $t, X_{t}$ is quasi-continuous in $\omega$, and for quasi every $\omega$, $t \rightarrow X_{t}(\omega)$ is continuous. Then if $\nu$ is a probability measure on $\Omega$ which does not charge polar sets and such that the process $\left(B_{t}\right)$ is an $\left(\mathcal{F}_{t}^{0}\right)$-semimartingale under $\nu$, the process $\tilde{X}_{t}$ is the solution of the same $\operatorname{SDE}$ under $\nu$, that is to say $\tilde{X}_{t}$ satisfies $\nu$-a.e.

$$
\forall t \quad \tilde{X}_{t}=x+\int_{0}^{t} \sigma\left(s, \tilde{X}_{s}\right) \stackrel{\nu}{\prime} d B_{s}+\int_{0}^{t} b\left(s, \tilde{X}_{s}\right) d s
$$

where $\int \sigma\left(s, \tilde{X}_{s}\right) \stackrel{\nu}{ } \cdot d B$, denotes the stochastic integral under $\nu$.

For a one dimensional Brownian motion $(d=1)$, the law of the Brownian bridge $\mathbb{E}\left[\cdot \mid B_{1}=a\right]$ is an example of such a measure $\nu$ which is singular with respect to the Wiener measure ( $\mathrm{cf}[\mathrm{B}-\mathrm{H} 4])$. For $d>1$ the same result is obtained by taking the conditional law of the Brownian motion given that $B_{1}$ belongs to an $(n-1)$-dimensional hyperplan with the Gauss measure on it.

B. The case which gives rise to a true extension of the classical stochastic calculus is when under $\nu\left(B_{t}\right)$ fails to be a semimartingale so that the right hand side has no direct meaning by itself.

We construct now a family of such measures on the Wiener space in the case $d=1$ for simplicity.

The idea is to consider a conditional law of the form $\mathbb{E}\left[\cdot \mid \int_{0}^{1} h_{0}(s) d B_{s}=0\right]$ for $h_{0} \in L^{2}([0,1]), \int_{0}^{1} h_{0}^{2}(s) d s=1$.

For using computations by decomposition on the Wiener chaos, we define this object as the positive measure which coincides on $\mathbb{D} \cap C(\Omega)$ with the distribution on the Wiener space

$$
\nu=\sqrt{2 \pi} \delta_{0}\left(\tilde{h_{0}}\right) \quad \tilde{h_{0}}=\int_{0}^{1} h_{0}(s) d B_{s}
$$


in the sense of Meyer-Yan [M2].

The characteristic functional of $\nu$ is

$$
U_{\nu}(\xi)=e^{-\frac{1}{2}<\xi, h_{0}>^{2}} \quad \xi \in C_{c}^{\infty}((0,1])
$$

so that its decomposition on the chaos is written

$$
\nu=\sum_{n} \frac{1}{n !} I_{n}\left(f_{n}\right)
$$

with

$$
\begin{cases}f_{n}=\frac{(2 p) !(-1)^{p}}{p ! 2^{p}} h_{0}^{\otimes 2 p} & \text { if } n=2 p \\ f_{n}=0 & \text { if } n=2 p+1\end{cases}
$$

where

$$
I_{n}(f)=n ! \int_{0<s_{1}<\ldots<s_{n}<1} f\left(s_{1}, \ldots, s_{n}\right) d B_{s_{1}} \ldots d B_{s_{n}}
$$

for symmetric $f \in L^{2}\left([0,1]^{n}\right)$.

So $\nu$ is a distribution of Watanabe and putting $\nu_{2 p}=\frac{1}{(2 p) !} I_{2 p}\left(f_{2 p}\right)$ one has

$$
\left\|\nu_{2 p}\right\|_{L^{2}}^{2}=\frac{(2 p) !}{(p !)^{2} 2^{2 p}}
$$

It follows that $\nu$ has a finite energy that is to say

$$
\|\nu\|_{2,-1}^{2}=\sum_{p=0}^{\infty} \frac{1}{1+2 p}\left\|\nu_{2 p}\right\|^{2} \leq 1+\sum_{p=1}^{\infty} \frac{1}{1+2 p} \cdot \frac{1}{\sqrt{e p}}<+\infty .
$$

Formula (4) extends to $\xi \in L^{2}([0,1])$

$$
<\nu, \mathcal{E}(\xi)>=e^{-\frac{1}{2}\left\langle\xi, h_{0}\right\rangle^{2}}
$$

where

$$
\mathcal{E}(\xi)=\exp \left[\int_{0}^{1} \xi_{s} d B_{s}-\frac{1}{2} \int_{0}^{1} \xi_{s}^{2} d s\right] .
$$

Let $h_{0}, h_{1}, \ldots, h_{n}, \ldots$ be a complete orthonormal system of $L^{2}([0,1])$, it follows from (6) that if $g$ is a polynomial one has

$$
<\nu, g\left(\tilde{h_{0}}, \tilde{h_{1}}, \ldots, \tilde{h_{n}}\right)>=<m, g\left(0, \tilde{h}_{1}, \ldots, \tilde{h_{n}}\right)>
$$

and $\nu$ is then a positive distribution of Watanabe hence $\nu$ is a measure ( $\mathrm{cf}[\mathrm{N}-\mathrm{U}]$ ).

For $t \in[0,1]$ we consider also the distrbutions $\nu_{t}$ defined by

$$
U_{\nu_{t}}(\xi)=\left\langle\nu, \mathcal{E}\left(\xi 1_{[0, t]}\right)>=e^{\left.-\frac{1}{2}<\xi, h_{0} 1_{[0, t]}\right\rangle^{2}} .\right.
$$

A similar computation as the previous one gives

$$
\left\|\nu_{t}\right\|_{2,-1} \leq\|\nu\|_{2,-1}
$$


The family $\left(\nu_{t}\right)$ is a distribution martingale in the sense of Yan [Y1] and the $\nu_{t}$ 's are probabilities on $\Omega$.

Following the notations of [Y1] we compute now the adapted projection of the distribution $D \nu$ where $D$ is the gradient operator. We have

$$
(D \nu)^{a d}(t)=\sum_{q=1}^{\infty} \frac{1}{(2 q-1) !} I_{2 q-1}\left(\left(h_{0} 1_{[0, t]}\right)^{\otimes 2 q-1} \frac{(2 q) !(-1)^{q}}{q ! 2^{q}} h_{0}(t)\right)
$$

and by using the formulae of Shigekawa [S1]

$$
\tilde{h} I_{n}\left(h^{\otimes n}\right)=I_{n+1}\left(h^{\otimes(n+1)}\right)+n\|h\|^{2} I_{n-1}\left(h^{\otimes(n-1)}\right)
$$

we obtain

$$
(D \nu)^{a d}(t)=1_{\{t<a\}} \frac{-h_{0}(t)}{1-\left\|h_{0} 1_{[0, t]}\right\|^{2}}\left(h_{0} \widetilde{1_{[0, t]}}\right) \cdot \nu_{t}
$$

where $\|\cdot\|$ is the norm of $L^{2}([0,1])$ and

$$
\left(\widetilde{h_{0} 1_{[0, t]}}\right)=\int_{0}^{t} h_{0}(s) d B_{s},
$$

and with

$$
a=\inf \left\{t: \int_{0}^{t} h_{0}^{2}(s) d s=1\right\} .
$$

If we write (8) in the following form

$$
(D \nu)^{a d}(t)=\zeta(t) \cdot \nu_{t}
$$

the formula of Ito-Ustunel [U1] gives

$$
\nu=m+\delta(\zeta(.) \nu .)
$$

From now on, we suppose $a<1$ and $h_{0}$ with bounded variation. $\left(\boldsymbol{h}_{0} \widetilde{1_{[0, t]}}\right)$ possesses then a version which is an $\left(\mathcal{F}_{t}^{0}\right)$-adapted process continuous in $(t, \omega)$ on $[0,1] \times \Omega$. In the sequel $\zeta$ is supposed to be defined from this version. (The following construction is also possible without supposing the variation of $h_{0}$ to be bounded. A version of $\left(\widetilde{h_{0} 1_{[0, t]}}\right)$ should be chosen continuous in $t$ and quasi-continuous in $\omega$, what is always possible).

That leads to the following lemma:

Lemma 4 . The process

$$
M_{t}=B_{t}-\int_{0}^{t} \zeta_{s} d s
$$

(where the integral is, for $t \geq a$, a semi-convergent integral) is an $\left(\mathcal{F}_{t}^{0}\right)$-Brownian motion under $\nu$. 
Proof. Using the fact that the measure $\nu$ restricted to the $\sigma$-field $\mathcal{F}_{t}^{0}$ is the measure $\nu_{t}$, we break up the interval $[0,1]$ in $[0, a),\{a\},(a, 1]$.

a) First if $t<a$, the distribution $\nu_{t}$ is a random variable in $L^{2}$, in other words the measure $\nu_{t}$ has a density in $L^{2}$ with respect to $m$.

Indeed setting $\theta=\int_{0}^{t} h_{0}^{2}(s) d s$,

$$
\begin{aligned}
\left\|\nu_{t}\right\|_{L^{2}}^{2} & =\sum_{p=0}^{\infty} \frac{(2 p) !(-1)^{p}}{p ! 2^{p}} \theta^{2 p} \\
& =\frac{1}{\sqrt{1-\theta^{2}}}<+\infty
\end{aligned}
$$

It follows then from (8) that if we set $n_{t}=\frac{d \nu_{t}}{d m}$ we have

$$
n_{t}=1+\int_{0}^{t} \zeta_{s} n_{s} d B_{s} \quad \text { for } t<a
$$

and because $\mathbb{P}\left[\int_{0}^{t} \zeta_{s}^{2} d s<+\infty\right]=1$ for $t<a$ we get

$$
n_{t}=\exp \left[\int_{0}^{t} \zeta_{s} d B_{s}-\frac{1}{2} \int_{0}^{t} \zeta_{s}^{2} d s\right]
$$

hence $n_{t}>0$ and in fact $\nu_{t}$ and $m$ are equivalent, $\nu_{t}$ being a probability it holds $\mathbb{E} n_{t}=1$ and the classical Girsanov theorem (cf [L1]) applies and gives the result. b) The study of the limit of $M_{t}$ for $t \uparrow a$ is obvious under $\nu$, and $M_{t}$ is an $\mathcal{F}_{t}^{0}$ Brownian motion under $\nu$ on $[0, a]$.

c) At last it follows easily from formula (7) that under $\nu \sigma$-fields $\mathcal{F}_{a}^{0}$ and $\sigma\left(B_{s}\right.$ $\left.B_{a}, a \leq s \leq 1\right)$ are independent and that

$$
<\nu, G F>=<\nu, G><m, F>
$$

if $\mathrm{G}$ is $\mathcal{F}_{a}^{0}$-measurable and if $F$ is $\sigma\left(B_{s}-B_{a}, a \leq s \leq 1\right)$-measurable, what gives the result by

$$
M_{t}=M_{a}+B_{t}-B_{a} \quad \text { for } t>a .
$$

Writing $B_{t}=M_{t}+\int_{0}^{t} \zeta_{s} d s$, we see that in order that the coordinates $\left(B_{t}\right)$ fail to be a semimartingale, it is sufficient to choose $h_{0}$ in such a way that the continuous process $\int_{0}^{t} \zeta_{s} d s$ fails to have a finite variation in the neighbourhood of $a$ under $\nu$.

As $m$ and $\nu$ are mutually singular, we must express $\zeta_{s}$ in terms of the Brownian motion $M_{t}$ under $\nu$.

Lemma 5 . For $t<a$, it holds

$$
\zeta_{t}=-h_{0}(t) \int_{0}^{t} \frac{h_{0}(s)}{\int_{s}^{a} h_{0}^{2}(u) d u} d M_{s}
$$


Proof. This comes from the fact that the relation $M_{t}=B_{t}-\int_{0}^{t} \zeta_{s} d s$ yields

$$
M_{t}=B_{t}+\int_{0}^{t}\left[\frac{h_{0}(s)}{\int_{s}^{a} h_{0}^{2}(u) d u} \int_{0}^{s} h_{0}(u) d B_{u}\right] d s
$$

and it is not difficult to see that this relation can be turned into the following

$$
M_{t}=B_{t}+\int_{0}^{t}\left[h_{0}(s) \int_{0}^{s} \frac{h_{0}(u)}{\int_{u}^{a} h_{0}^{2}(v) d v} d M_{u}\right] d s .
$$

To show that it is possible to choose $h_{0}$ in such a way that

$$
I:=\int_{0}^{a}\left|\zeta_{s}\right| d s=+\infty \quad \nu-\text { a.s. }
$$

we perform some transformations:

Let $u$ be a function from $[0, \infty)$ into $(0, \infty)$ such that

$$
\int_{0}^{\infty} \frac{u^{2}(t)}{t+1} d t=a
$$

The map $y \rightarrow a-\int_{y}^{\infty} \frac{u^{2}(t)}{t+1} d t$ being stricly increasing, we can define a function $\xi:[0, a) \rightarrow[0, \infty)$ by

$$
\forall s \in[0, a) \quad a-\int_{\xi(s)}^{\infty} \frac{u^{2}(t)}{t+1} d t=s .
$$

Then if we set

$$
h_{0}(s)=\frac{\sqrt{\xi^{\prime}(s)}}{\xi(s)+1}
$$

it holds $\int_{0}^{a} h_{0}^{2}(t) d t=1$ and $\int_{s}^{a} h_{0}^{2}(t) d t=\frac{1}{\xi(s)+1}$ hence

$$
\xi(s)=\frac{\int_{0}^{s} h_{0}^{2}(u) d u}{\int_{s}^{a} h_{0}^{2}(u) d u} .
$$

But the process

$$
Y_{t}=\int_{0}^{t} \frac{h_{0}(s)}{\int_{s}^{a} h_{0}^{2}(u) d u} d M_{s}
$$

is a continuous martingale with bracket

$$
<Y, Y>_{t}=\frac{\int_{0}^{t} h_{0}^{2}(u) d u}{\int_{t}^{a} h_{0}^{2}(u) d u} .
$$


Therefore there exists a Brownian motion $\left(W_{t}\right)$ such that $Y_{t}=W_{\xi(t)}$ and the integral to be studied can be written

$$
\begin{aligned}
I & =\int_{0}^{a}\left|h_{0}(t)\right|\left|W_{\xi(t)}\right| d t \\
& =\int_{0}^{\infty} \frac{\left|h_{0}\left(\xi^{-1}(s)\right)\right|}{\xi^{\prime}\left(\xi^{-1}(s)\right)}\left|W_{s}\right| d s .
\end{aligned}
$$

Since by (12) it holds $\xi^{\prime}\left(\xi^{-1}(s)\right)=\frac{s+1}{u^{2}(s)}$ one gets with (13)

$$
I=\int_{0}^{\infty} \frac{u(s)}{(s+1)^{3 / 2}}\left|W_{s}\right| d s
$$

Hence it is enough to find a function $u>0$ satisfying (11) and such that in (14) one gets $+\infty$. For this we use the following version of a lemma of Jeulin [J1]:

Lemma 6 . Let $R_{t}$ be a positive measurable real process on a probability space $(\Omega, \mathbb{P})$ such that

1) the law $\nu$ of $R_{t}$ does not depend on $t$

2) $\nu(\{0\})=0$

3) $\int x d \nu(x)<+\infty$

then for any positive Radon measure $\mu$ on $\mathbb{R}_{+}$

$$
\begin{aligned}
& \text { i) } \quad \int_{0}^{\infty} d \mu(t)<+\infty \Rightarrow \quad \int_{0}^{\infty} R_{t} d \mu(t) \in L^{1}(\mathbb{P}) \\
& \text { ii) } \int_{0}^{\infty} d \mu(t)=+\infty \Rightarrow \int_{0}^{\infty} R_{t} d \mu(t)=+\infty \quad \mathbb{P a} \text { a.s. }
\end{aligned}
$$

Proof. The point i) is clear because

$$
\mathbb{E} R_{t}=\int x d \nu(x)<+\infty
$$

For the second point let $n \in \mathbb{N}$ and $J_{n}=\left\{\int_{0}^{\infty} R_{t} d \mu(t) \leq n\right\}$. Suppose $\mathbb{P}\left(J_{n}\right)>0$, then

$$
\begin{aligned}
\mathbb{E}\left[1_{J_{n}} R_{t}\right] & =\int_{0}^{\infty} d u \mathbb{E}\left[1_{J_{n}} 1_{\left\{R_{t}>u\right\}}\right]=\int_{0}^{\infty} d u \mathbb{E}\left[\left(1_{J_{n}}-1_{\left\{R_{t} \leq u\right\}}\right)^{+}\right] \\
& \geq \int_{0}^{\infty} d u\left(\mathbb{P}\left(J_{n}\right)-\nu([0, u])\right)^{+}
\end{aligned}
$$

and by the hypothesis 2$) \lim _{u \rightarrow 0} \nu([0, u])=0$, hence the last integral is equal to $a_{n}>0$. By integration

$$
n \mathbb{P}\left(J_{n}\right) \geq a_{n} \int_{0}^{\infty} d \mu(t)
$$

what gives ii) by contraposition.

It follows by taking $R_{t}=\frac{\left|W_{t}\right|}{\sqrt{t}}$ that $I=+\infty$ as soon as

$$
\int_{0}^{\infty} \frac{u(s) \sqrt{s}}{(s+1)^{3 / 2}} d s=+\infty
$$


There are several functions satifying (11) and (15), for example $u(s)=1 /\left(\frac{1}{a}+\right.$ $\log (s+1))$, which gives

$$
h_{0}(t)=\frac{e^{\frac{1}{2 a}-\frac{1}{2(a-t)}}}{a-t} 1_{[0, a)}(t) .
$$

Let us summarize the preceding discussion. Let $h_{0}$ associated with $u$ by (12) and (13) and let $\nu$ be the distribution on the Wiener space associated with $h_{0}$ by (3) and (4). $\nu$ is a distribution of Watanabe in $\mathbb{D}_{2,-1}$ and is also a positive measure which does not charge polar sets.

For $t<a$, on the $\sigma$-field $\mathcal{F}_{t}^{0}$ the measures $m$ and $\nu$ are equivalent, $\left(B_{s}\right)_{s \leq t}$ is an $\left(\mathcal{F}_{t}^{0}\right)$-Brownian motion under $m$ and an $\left(\mathcal{F}_{t}^{0}\right)$-semimartingale under $\nu$.

For $t \geq a$, the measures $m$ and $\nu$ are mutually singular on the $\sigma$-field $\mathcal{F}_{t}^{0}$, $\left(B_{s}\right)_{s \leq t}$ is not an $\left(\mathcal{F}_{t}^{0}\right)$-semimartingale under $\nu$, nevertheless the process

$$
B_{s}-\int_{0}^{a}\left[\frac{h_{0}(v)}{\int_{v}^{a} h_{0}^{2}(u) d u} \int_{0}^{v} h_{0}(u) d B_{u}\right] d v
$$

is an $\left(\mathcal{F}_{s}^{0}\right)$-Brownian motion under $\nu$.

It is possible to build examples of measures which do not charge polar sets and for which the singularity which is here at the point $a$, appears along a whole interval. Such measures are solutions in sense of distributions of Watanabe of the stochastic differential equation which defines the exponential of Doléans. This will be published elsewhere.

\section{References}

[B1] N. Bouleau, Désintégration des mesures d'énergie dans les espaces de Dirichlet et propriété de densité de temps d'occupation. C. R. Acad. Sc. Paris, t298, sI, $\mathrm{n} 7,1984$.

[B-H1] N. Bouleau, F. Hirsch. Formes de Dirichlet générales et densité des variables aléatoires réelles sur l'espace de Wiener. J. of Func. Analysis 69, $n^{\circ} 2,229-259$, 1986.

[B-H2] N. Bouleau, F. Hirsch. Propriétés d'absolue continuité dans les espaces de Dirichlet et application aux équations différentielles stochastiques. Seminaire Prob XX, Lect. Notes in Math.1204, Springer 1985.

[B-H3] N. Bouleau, F. Hirsch. On the derivability, with respect to the initial data, of the solution of a stochastic differential equation with Lipschitz coefficients. Seminaire Th. du Potentiel n9, Springer 1989. 
[B-H4] N. Bouleau, F. Hirsch. Quasi-everywhere regularity of solutions of Brownian stochastic differential equations and their flows. Journal de Math. Pures et Appliquées (to appear).

[F1] H. Federer. Geometric Measure Theory, Springer 1969.

[F2] D. Feyel, A. de la Pradelle. Espaces de Sobolev gaussiens, Ann. Inst. Fourier (to appear)

[F3] M. Fukushima. Basic properties of Brownian motion and a capacity on the Wiener space, J. Math. Soc. Japan 36,1,161-175,1984.

[J1] Th. Jeulin. Semi-martingales et grossissement de filtrations, Lect. Notes in Math. 333, Springer 1980.

[K1] T. Komatsu,K. Takashima. The Hausdorff dimension of quasi-all Brownian paths, Osaka J. Math., 21, 613-619,1984. On the existence of intersectional local time except on zero capacity set, id., 913-929.

[K2] H. Kunita. Stochastic differential equations and stochastic flows of diffeomorphisms, in Ec. d'été de St Flour XII, 1982, Lect. Notes in Math. 1097, Springer 1984.

[L1] R.S. Liptser, A.N. Shiryayev. Statistic of random processes I general theory, Springer 1977.

[N-U] D. Nualart, S. Ustunel. Mesures cylindriques et distributions sur l'espace de Wiener. Proc. Trento (to appear)

[M1] P. Malliavin. Implicit functions of finite corank on the Wiener space, in proc. Tanigushi symp.1982,369-386, Kinokuniya, 1983.

[M2] P.A. Meyer, J.A. Yan. A propos des distributions sur l'espace de Wiener, Seminaire Prob. XXI, Lect. Notes in Math. 1247, Springer 1987.

[O1] D. Ocone. A guide to stochastic calculus of variations, proc. Silivri 1986, Lect. Notes in Math. 1316, Springer 1988.

[R1] J. Ren. Thèse de doctorat de l'Université Paris VI, 1988.

[S1] I. Shigekawa. Derivatives of Wiener functionals and absolute continuity of induced measures, J. Math. Kyoto Univ., 20-2, 263-289, 1980.

[S2] I. Shigekawa. On quasi-everywhere existence of local time of 1-dimensional Brownian motion, Osaka J. Math., 21, 621-627, 1984. 
[U1] S. Ustunel. Representation of the distributions on the Wiener space and stochastic calculus of variations, J. funct. Anal., 70, 126-139, 1987.

[W1] S. Watanabe. Lectures on stochastic differential equations and Malliavin calculus, Tata Institute, Bombay 1984.

[Y1] J.A. Yan. Développement des distributions sur les chaos de Wiener et application à l'analyse stochastique, Seminaire Prob. XXI, Lect. Notes in Math. 1247, Springer 1987. 